\title{
Optical properties of MgZnO alloys: Excitons and exciton-phonon complexes
}

\author{
M. D. Neumann, ${ }^{1, \text { a) }}$ C. Cobet, ${ }^{1}$ N. Esser, ${ }^{1}$ B. Laumer, ${ }^{2,3}$ T. A. Wassner, ${ }^{3}$ M. Eickhoff, ${ }^{2}$ \\ M. Feneberg, ${ }^{4}$ and R. Goldhahn ${ }^{4}$ \\ ${ }^{1}$ Leibniz-Institut für Analytische Wissenschaften - ISAS - e.V., Albert-Einstein-Str. 9, 12489 Berlin, Germany \\ ${ }^{2}$ I. Physikalisches Institut, Justus-Liebig-Universität Giessen, Heinrich-Buff-Ring 16, 35392 Giessen, Germany \\ ${ }^{3}$ Walter Schottky Institut, Technische Universität München, Am Coulombwall 3, 85748 Garching, Germany \\ ${ }^{4}$ Institut für Experimentelle Physik, Otto-von-Guericke-Universität Magdeburg, 39106 Magdeburg, Germany
}

(Received 4 March 2011; accepted 27 May 2011; published online 11 July 2011)

\begin{abstract}
The characteristics of the excitonic absorption and emission around the fundamental bandgap of wurtzite $\mathrm{Mg}_{x} \mathrm{Zn}_{1-x} \mathrm{O}$ grown on c-plane sapphire substrates by plasma assisted molecular beam epitaxy with $\mathrm{Mg}$ contents between $x=0$ and $x=0.23$ are studied using spectroscopic ellipsometry and photoluminescence (PL) measurements. The ellipsometric data were analyzed using a multilayer model yielding the dielectric function (DF). The imaginary part of the DF for the alloys exhibits a pronounced feature which is attributed to exciton-phonon coupling (EPC) similar to the previously reported results for $\mathrm{ZnO}$. Thus, in order to determine reliable transition energies, the spectral dependence is analyzed by a model which includes free excitonic lines, the exciton continuum, and the enhanced absorption due to EPC. A line shape analysis of the temperature-dependent PL spectra yielded in particular the emission-related free excitonic transition energies, which are compared to the results from the DF line-shape analysis. The PL linewidth is discussed within the framework of an alloy disorder model. (C) 2011 American Institute of Physics. [doi:10.1063/1.3606414]
\end{abstract}

\section{INTRODUCTION}

Zinc oxide $(\mathrm{ZnO})$ with its room temperature bandgap of around $3.4 \mathrm{eV}$ (Ref. 1) is considered as a promising material for optoelectronic devices operating in the violet and ultraviolet regime. The large exciton binding energy close to $60 \mathrm{meV}$ (Ref. 2) leads to pronounced excitonic effects in emission and absorption spectra up to room temperature. It paves the way to device operation based on excitonic interaction, e.g., cavity-coupled polariton lasers. ${ }^{3}$ As the key structure for optoelectronic devices, the realization of heterojunctions to facilitate, for example, the confinement of charge carriers and photons, is a basic requirement. Ternary $\mathrm{Mg}_{x} \mathrm{Zn}_{1-x} \mathrm{O}$ alloys have been shown to be a suitable material for this purpose, allowing bandgap tuning toward higher energies. ${ }^{4}$ In order to make use of these materials and to allow device optimization, precise knowledge of the optical and band structure properties around the fundamental absorption edge is a precondition.

The complex dielectric function $(\mathrm{DF}) \varepsilon=\varepsilon_{1}+\mathrm{i} \varepsilon_{2}$, which relates the dielectric displacement and the electric field of the electro-magnetic wave, reflects not only the band structure peculiarities but also many-particle interactions. Electron-hole interaction (exciton effect) in $\mathrm{ZnO}$ leads to an appreciable change of the DF with respect to the single-particle picture. It causes peak shifts and redistribution of oscillator strength in the vacuum-ultraviolet spectral range. ${ }^{5,6}$ The behavior around the absorption edge appears to be even more complicated. To analyze the DF considering excitonic effects the approach presented by Elliott ${ }^{7}$ is commonly used. How-

\footnotetext{
a) Author to whom correspondence should be addressed. Electronic mail: maciej.neumann@isas.de.
}

ever, the experimental studies of Jellison and Boatner ${ }^{8}$ revealed additional features in the room temperature DF around the bandgap that could not be described by Elliott's theory alone. Such features of the optical properties had been known before. Studying the transmission properties of $\mathrm{ZnO}$, Liang and Yoffe $^{9}$ observed sidebands separated about one LO-phonon energy above the main excitonic peak and attributed them to exciton-phonon bound states or so called exciton-phonon complexes (EPC). A recently published highresolution $\mathrm{DF}$ of $\mathrm{ZnO}$ at low temperature ${ }^{10}$ exhibits the sidebands as well, but they where not discussed further. These features are not only observed in $\mathrm{ZnO}$, but have also been found in optical spectra of many partly ionic materials. ${ }^{1-14}$

Theoretical considerations of EPC contributions to the DF can be found in Refs. 15-19. In recent years only two slightly different phenomenological models by Shokhovets et $a l .{ }^{18}$ and Müller et al. ${ }^{19}$ became widely accepted. Both account for the EPC as phonon replicas of the discrete excitonic contribution. Shokhovets analyzed the imaginary part of the DF of $\mathrm{ZnO}$ at room temperature, showing that EPC introduce significant contributions. Müller et al., on the other hand, investigated $\mathrm{MgZnO}$ films at liquid $\mathrm{He}$ temperature using transmission measurements and showed that the absorption coefficient exhibits clear signatures attributed to EPC. The many-particle effect was taken into account for determining more reliable exciton transition energies. Other studies determined and analyzed the room-temperature DF of $\mathrm{MgZnO} ;{ }^{20}$ however, the EPC contributions had not been incorporated in the model; an apparently related feature in the imaginary part of the DF was interpreted as an excited excitonic state.

It turns out that the combined influence of electron-hole interactions and $\mathrm{EPC}$ on the $\mathrm{DF}$ of $\mathrm{MgZnO}$ alloys has not 
been investigated so far. It is the subject of the current paper. The application of spectroscopic ellipsometry (SE) allows the determination of the DF without making any assumptions concerning their shape. These data are then analyzed by employing the parametric DF model which includes both the excitons and exciton-phonon complexes. The fit yields characteristic energies, in particular the free excitonic transition energies and the average phonon energies of the EPC. Using temperature-dependent photoluminescence measurements, the associated transition energies of the exciton recombinations can be traced back up to room temperature and are compared to the results from SE.

\section{SAMPLES AND EXPERIMENTAL DETAILS}

Plasma-assisted molecular beam epitaxy was employed for growth of (0001)-oriented c-plane wurtzite $\mathrm{Mg}_{x} \mathrm{Zn}_{1-x} \mathrm{O}$ layers on c-plane sapphire substrates. Indium was used to mount the samples on the substrate holder. In the initial stage of growth a thin layer of $\mathrm{MgO}$ was deposited at a substrate temperature of $600^{\circ} \mathrm{C}$, followed by a thin layer of $\mathrm{ZnO}$ at $300^{\circ} \mathrm{C}$. Subsequent annealing led to a smooth surface morphology and intermixing of the two layers. ${ }^{21,22}$ Then, the $\mathrm{MgZnO}$ layers under consideration were grown on this $\mathrm{MgO} / \mathrm{ZnO}$ double-buffer according to the growth process described in Ref. 22. In the present study two different series of samples were investigated: The first was grown under stoichiometric growth conditions at a substrate temperature of $500{ }^{\circ} \mathrm{C}$ with a $\mathrm{Mg}$ content $x$ up to 0.06 and an average layer thickness of $300 \mathrm{~nm}$, referred to as high temperature (HT) series. For the high temperature growth an increase of the $\mathrm{Mg}$ content above $6 \%$ was inhibited by the occurrence of phase separation into wurtzite $\mathrm{Zn}(\mathrm{Mg}) \mathrm{O}$ and cubic $\mathrm{Mg}(\mathrm{Zn}) \mathrm{O}$, as found by X-ray diffraction (XRD). Therefore, three samples were grown at a lower substrate temperature of $270^{\circ} \mathrm{C}$ and metal rich growth conditions, by which a $\mathrm{Mg}$ content of $23 \%$ could be achieved. This second series is referred to as the low temperature (LT) series and has layer thicknesses of around $1000 \mathrm{~nm}$. The thicknesses and $\mathrm{Mg}$ contents were determined by ellipsometry and secondary ion mass spectroscopy measurements, respectively.

The structural properties of the $\mathrm{MgZnO}$ layers studied in the current work by optical methods were investigated by high resolution X-ray diffraction, revealing no indication for cubic $\mathrm{MgO}$ phases ( $2 \Theta-\omega$ scans, not shown here). Reciprocal space maps were recorded around the asymmetric (205) reflex for determination of the $a$ - and $c$-lattice parameters depicted in Fig. 1 as a function of the Mg content. The $a$-lattice parameter slightly increases with increasing $\mathrm{Mg}$ content, while the $c$-lattice parameter decreases. Though the absolute values differ in magnitude by the order of $10^{-3} \AA$, the observed dependence on the $\mathrm{Mg}$ content is in agreement with XRD measurements ${ }^{4}$ and DFT calculations ${ }^{23,24}$ found in former publications, indicating relaxation for the thick samples of the LT series.

As a result of the weak variation of the lattice parameters with $\mathrm{Mg}$, the strain state of the thin samples from the HT series with $\mathrm{Mg}$ concentrations $x \leq 0.06$ cannot be finally concluded from the XRD results alone, i.e., gradual relaxa-

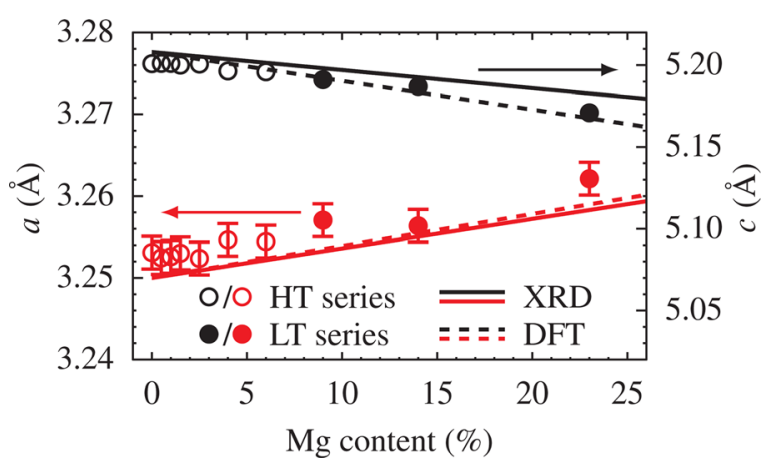

FIG. 1. (Color online) Lattice parameters of the $\mathrm{MgZnO}$ layers extracted from reciprocal space maps around the (205) reflex (circles) in comparison to literature values from XRD measurements (Ref. 4) and DFT calculations (Ref. 23) (the values for the $a$ parameter of Ref. 23 are shifted by $8.3 \mathrm{pm}$ to smaller values, while those for the $c$ parameter are shifted by $10.1 \mathrm{pm}$ to higher values for better agreement with the XRD values).

tion along the growth direction might occur. The low-temperature PL data of $\mathrm{ZnO}$ discussed below provide a higher accuracy in this respect. They indicate strain-free material within the light penetration depth.

The ellipsometry measurements were performed at room temperature (RT) in a photon energy range from 1.2 to $5 \mathrm{eV}$ with a spectral resolution of $10 \mathrm{meV}$ at angles of incidence of $62^{\circ}, 68^{\circ}$, and $74^{\circ}$ using a commercial rotating-analyzer ellipsometer with auto retarder function (Woollam Inc., VASE model).

The photoluminescence (PL) investigations were conducted around the bandgap regions using the $325 \mathrm{~nm}$ (3.81 $\mathrm{eV}$ ) line of a $\mathrm{HeCd}$ laser for excitation, a double-grating monochromator with $850 \mathrm{~mm}$ focal length as the dispersive element, and a liquid $\mathrm{N}_{2}$-cooled charge coupled device sensor to detect the signal. The spectral resolution amounts to $0.3 \mathrm{meV}$ at around $3.5 \mathrm{eV}$. The samples were mounted into a helium bath cryostat with tunable temperature between $5 \mathrm{~K}$ and $295 \mathrm{~K}$. Due to the limited excitation energy the $x=0.23$ sample was not investigated by PL.

\section{THEORY}

\section{A. Ellipsometric data analysis}

Spectroscopic ellipsometry determines the relative change of the polarization state of light due to reflection. This is expressed in terms of the ellipsometric parameters $\Psi$ and $\Delta$, representing the change in magnitude and phase, respectively. For isotropic or uniaxial samples with (0001)orientation, the off-diagonal elements of the Jones matrix vanish, ${ }^{25}$ i.e., no transformation from $p$ - into $s$-polarized light or vice versa occurs. Then, ellipsometry yields only $\Psi_{p p}=\Psi$ and $\Delta_{p p}=\Delta$ which are related to the complex reflection coefficients $\bar{r}_{p}$ and $\bar{r}_{s}$ by

$$
\frac{\bar{r}_{p}}{\bar{r}_{s}}=\tan \Psi e^{i \Delta} .
$$

$\mathrm{MgZnO}$ alloys in wurtzite structure are optically anisotropic materials as expressed by the ordinary $\left(\bar{\varepsilon}_{\perp}\right)$ and extraordinary $\left(\bar{\varepsilon}_{\|}\right)$DFs corresponding to electric field polarization perpendicular $(\mathbf{E} \perp \mathbf{c})$ and parallel $(\mathbf{E} \| \mathbf{c})$ to the optical axis, 
respectively. A parameter-free determination of both tensor components requires measurements on a- or m-plane surfaces for which the c-axis can be oriented either parallel or perpendicular to the plane of incidence. Such studies ${ }^{8,26}$ of $\mathrm{ZnO}$ bulk crystals revealed a pronounced shift of the extraordinary absorption edge toward higher photon energies with respect to the ordinary one. For (0001)-oriented material, $\Psi$ and $\Delta$ mainly depend on $\bar{\varepsilon}_{\perp}$ for energies close to the bandgap energy, while the contribution of $\bar{\varepsilon}_{\|}$is weak. It was demonstrated in Ref. 26 that a so-called isotropic fit, which disregards the anisotropy, yields a DF similar to the ordinary DF. Its analysis yields characteristic transition energies (free excitons and EPC) which are not shifted with respect to the data obtained from $\bar{\varepsilon}_{\perp}$. Only a weak enhancement of the magnitude of the free excitonic contribution is observed. Furthermore, Aspnes ${ }^{27}$ showed that the extraordinary DF has a minor influence on $\Psi$ and $\Delta$, far above the absorption edge for this geometry. Together with the small birefringence ${ }^{8}$ it is a sufficient approach, at least for small $\mathrm{Mg}$ contents, to treat the present samples as an isotropic material, i.e., the isotropically fitted DF is close to $\bar{\varepsilon}_{\perp}$.

The multilayer structure of the samples investigated here introduces interference and multiple reflections at each interface. This prevents a direct calculation of the DF from the experimentally obtained $\Psi$ and $\Delta$ within the two-phase model. Therefore the $\mathrm{DF}$ of the $\mathrm{MgZnO}$ layer is obtained by a multilayer fitting procedure of $\Psi$ and $\Delta$ similar to Ref. 28 , including substrate, buffer, $\mathrm{MgZnO}$, and surface roughness. The DF of the sapphire substrate is taken from Ref. 29. The DF for the buffer is obtained by a preceding analysis of substrates with a bare buffer layer of 15 to $20 \mathrm{~nm}$ thickness. The shift of the absorption edge toward higher energies in comparison to $\mathrm{ZnO}$ indicates a $\mathrm{Mg}$-content of 2-3\% in the buffer. The surface roughness ( 3 to $6 \mathrm{~nm}$ overlayer thickness) was taken into account by a Bruggeman effective medium approximation. ${ }^{30}$ Finally, no assumption was made concerning the shape of the MgZnO DFs, which means that $\varepsilon_{1}$ and $\varepsilon_{2}$ were fitted separately for each photon energy, yielding the so-called point-by-point (PbP) DFs.

\section{B. Model dielectric function}

In the second step, the imaginary part of the $\mathrm{DF} \varepsilon_{2}$ of the $\mathrm{MgZnO}$ films is analyzed on the basis of the PbP data and in the vicinity of the absorption edge using a parametric model DF which comprises three parts: contributions of free excitons, the exciton continuum, and the EPC. As pointed out before, $\varepsilon_{2}$ is similar to the ordinary DF. Owing to the valence band (VB) ordering $\Gamma_{7}-\Gamma_{9}-\Gamma_{7}$ (Refs. 1, 10 and 31) and the relative oscillator strengths, ${ }^{32}$ only contributions from discrete $\mathrm{A}$ and $\mathrm{B}$ excitons (denoted as $\mathrm{FX}_{\mathrm{A}}$ and $\mathrm{FX}_{\mathrm{B}}$ ) and their related EPC and exciton continua determine the optical response for $\mathbf{E} \perp \mathbf{c}$. The splitting between $\mathrm{FX}_{\mathrm{A}}$ and $\mathrm{FX}_{\mathrm{B}}$ amounts to $\sim 5.5 \mathrm{meV}$ for $\mathrm{ZnO}$ and thus can be resolved in low-temperature spectra, ${ }^{10}$ but not in a room-temperature DF (Refs. 8, 18 and 26) to be analyzed here. Furthermore, no detailed experimental studies on the VB splitting for hexagonal $\mathrm{MgZnO}$ or $\mathrm{MgO}$ have been reported so far due to the lack of strain-free nonpolar material. Recent band-structure calculations $^{33,34}$ revealed a VB ordering of $\Gamma_{7}-\Gamma_{9}-\Gamma_{7}$ also for wurtzite $\mathrm{MgO}$. The obtained results for the spin-orbit energy indicate that the splitting between $\mathrm{FX}_{\mathrm{A}}$ and $\mathrm{FX}_{\mathrm{B}}$ should decrease from $\mathrm{ZnO}$ toward $\mathrm{MgO}$ in the compositional range addressed here.

Due to this low splitting it is reasonable to replace in the $\mathrm{DF}$ data analysis $\mathrm{FX}_{\mathrm{A}}$ and $\mathrm{FX}_{\mathrm{B}}$ by a single discrete excitonic resonance $\mathrm{FX}_{\mathrm{X}}^{\mathrm{SE}}$ below the gap $E_{G}$, characterized by an average exciton binding energy of $E_{\mathrm{B}}^{\mathrm{X}}$. It causes an inaccuracy of less than $3 \mathrm{meV}$ for the determined characteristic energies.

For determination of the excitonic lines and the exciton continuum, Elliott's model ${ }^{7}$ is used, including a Gaussian broadening described by the parameter $\gamma$. With the energy gap $E_{\mathrm{G}}$, contributions to $\varepsilon_{2}$ from discrete exciton states with the exciton binding energy $E_{\mathrm{B}}^{\mathrm{X}}$ add up to

$$
\varepsilon_{2}^{X}=\frac{C^{X}}{(\hbar \omega)^{2}} \sum_{n} \frac{1}{\gamma n^{3}} \exp \left(-\frac{(\hbar \omega-E)^{2}}{\gamma^{2}}\right)
$$

where $E=E_{\mathrm{G}}-E_{\mathrm{B}}^{\mathrm{X}} / n^{2}$, with $n$ being the exciton state. The contribution from the exciton continuum results in

$$
\varepsilon_{2}^{\mathrm{BB}}=\frac{C^{\mathrm{BB}}}{(\hbar \omega)^{2}} \frac{1+\operatorname{erf}\left(\frac{\hbar \omega-E_{\mathrm{G}}}{\gamma}\right)}{1-\exp (-2 \xi)}
$$

where $\xi^{2}=\pi^{2}\left|E_{\mathrm{B}}^{\mathrm{X}} /\left(\hbar \omega-E_{\mathrm{G}}\right)\right|$. The parameters $C^{\mathrm{X}}$ and $C^{\mathrm{BB}}$ are proportional to the transition matrix elements ${ }^{32}$ and define the intensity of the transitions.

For the EPC contribution the approach of Shokhovets ${ }^{18}$ is used. Following the polaron picture of charge carriers in ionic crystals, being described by an accompanying phonon cloud, EPC are modeled as multi-phonon replicas of the excitonic contribution $\varepsilon_{2}^{\mathrm{X}}$ shifted by $m=1,2,3, \ldots$ times the average phonon energy $\Delta E_{\mathrm{Ph}}$. Using $E=E_{\mathrm{G}}-E_{\mathrm{B}}^{\mathrm{X}} / n^{2}$ $+m \Delta E_{\mathrm{Ph}}$, the EPC contribution reads

$$
\varepsilon_{2}^{\mathrm{EPC}}=f_{0} \sum_{m} b^{m-1} \varepsilon_{2}^{\mathrm{X}}
$$

The two scaling factors can be understood as a coupling factor $f_{0}$ and a cross section factor $b^{m-1}$, the latter being a measure of the probability of occurrence of the $m$ th phonon state. The quantity $F^{\mathrm{EPC}}=f_{0} \sum_{m} b^{m-1}$ then expresses the strength of the EPC contribution as compared to that of the discrete exciton.

\section{RESULTS AND DISCUSSION}

\section{A. Dielectric function at room temperature}

Figures 2(a) and 2(b) show the recorded spectra of the ellipsometric parameters $\Psi$ and $\Delta$ for the $\mathrm{Mg}_{0.06} \mathrm{Zn}_{0.94} \mathrm{O}$ sample. Using the multi-layer analysis described in Sec. III A, the RT PbP DF depicted in Fig. 2(c) is obtained. Although the data for $\Psi$ and $\Delta$ at low photon energies are strongly influenced by Fabry-Pérot-like oscillations, a smooth curve for $\varepsilon_{1}$ is obtained in this range. It emphasizes the accuracy of the multi-layer approach, as otherwise residual oscillations would appear. As expected for a low Mg content of only 6\%, the spectral dependence of the DF is similar to the results 

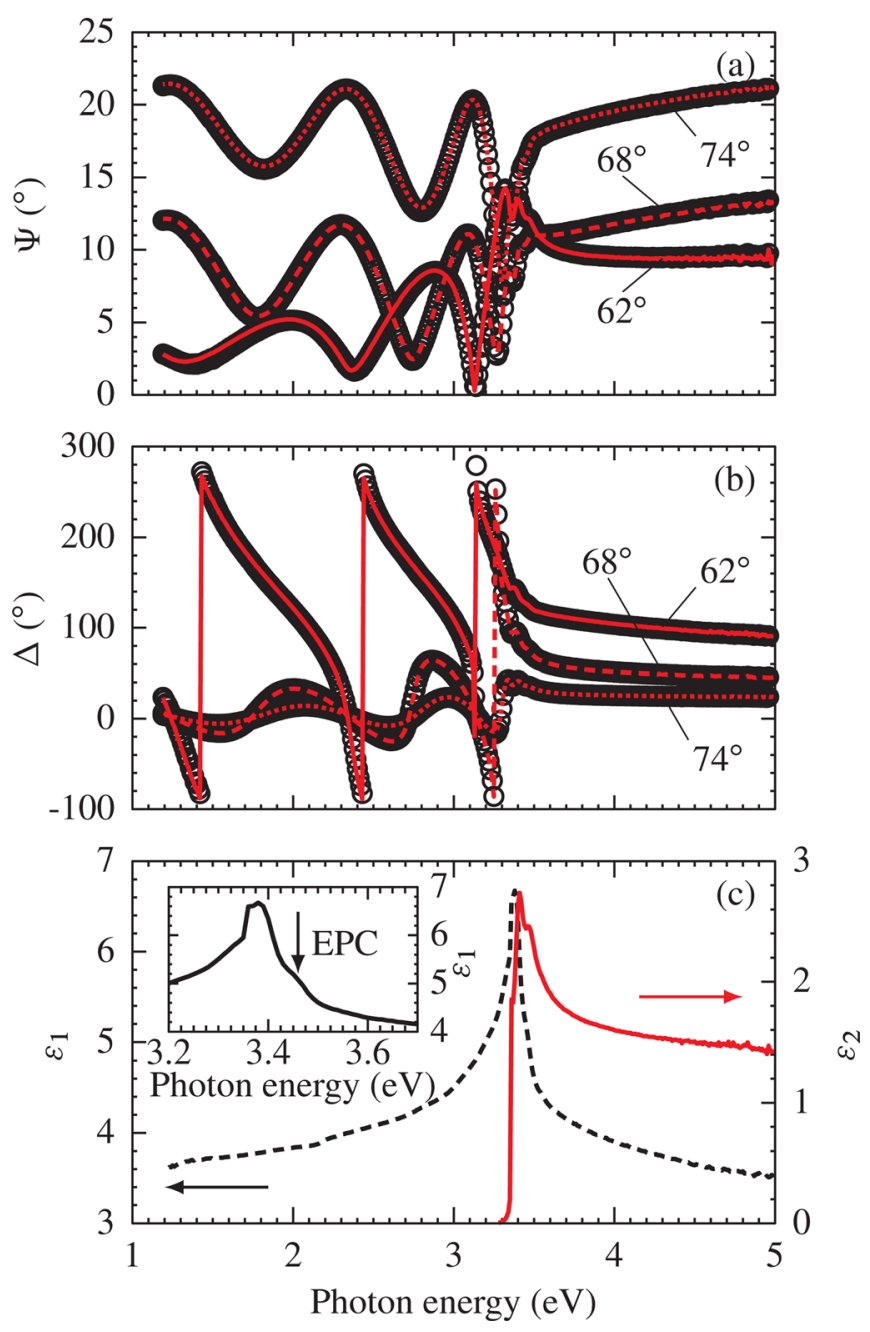

FIG. 2. (Color online) Ellipsometric parameters $\Psi$ (a) and $\Delta$ (b) of the $\mathrm{Mg}_{0.06} \mathrm{Zn}_{0.94} \mathrm{O}$ sample for different angles of incidence, and (c) real $\left(\varepsilon_{1}\right)$ and imaginary $\left(\varepsilon_{2}\right)$ part of the extracted complex DF at room temperature. Experimental data and point-by-point fits are shown as circles and lines, respectively. The inset in (c) shows a magnification of $\varepsilon_{1}$ in the bandgap region, with an arrow emphasizing the impact of EPC.

obtained for $\mathrm{ZnO} .^{8,26}$ The imaginary part exhibits a sharp onset at the absorption edge with a peak at $3.41 \mathrm{eV}$ followed by a shoulder (EPC) about $60 \mathrm{meV}$ higher in energy and a continuously decreasing value toward higher energies. The inset of Fig. 2(c) shows a magnification of $\varepsilon_{1}$ around the absorption edge; the shoulder attributed to EPC is clearly visible. A summary of $\varepsilon_{2}$ for all samples compared to the PL data, to be discussed below, is presented in Fig. 3. The line shape of the imaginary part of the HT series up to $x=0.06$ is very similar, with a sharp onset followed by a plateau. The LT series exhibits a much stronger broadening of the absorption edge.

Figure 4 demonstrates the influence of EPC on the optical response in more detail, the samples with $x=0.06$ and $x=0.01$ are chosen as typical examples. If EPC is disregarded in the first step, i.e., only a discrete excitonic transition and the continuum according to Eqs. (2) and (3) are used, then the solid line in Fig. 4(a) is obtained as a result of the fit for the $\mathrm{PbP} \varepsilon_{2}$ data of $\mathrm{Mg}_{0.06} \mathrm{Zn}_{0.94} \mathrm{O}$ around the bandgap. The following parameters were determined by the fitting procedure: bandgap $E_{\mathrm{G}}$, exciton binding energy $E_{\mathrm{B}}^{\mathrm{X}}$,

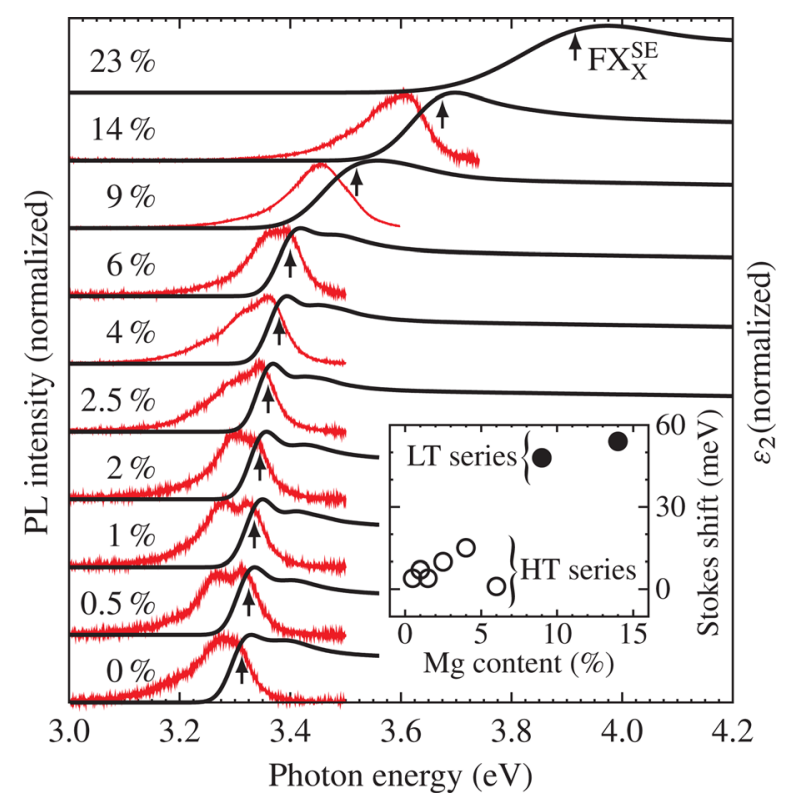

FIG. 3. (Color online) Comparison of the normalized room temperature spectra obtained by PL (thick, red) and SE (thin, black), respectively. The arrows show the position of the free exciton found from SE. The inset depicts the corresponding Stokes shift for the high (HT) and low (LT) temperature series.

and the intensity parameters $C^{\mathrm{X}}$ and $C^{\mathrm{BB}}$. The transition energy of the exciton line coincides with the peak in $\varepsilon_{2}$. A strong discrepancy between the $\mathrm{PbP}$ data and the model is found at the shoulder, slightly above the bandgap energy $E_{G}$. Due to its shape, size, and position this deviation cannot originate in excited exciton states, (which are already included), or in the $\mathrm{C}$ exciton and is assigned to EPC, as described in Ref. 18.

Figure 4(b) shows the corresponding result if the EPC contribution is additionally taken into account according to Eq. (4) with the fit parameters of average phonon energy $\Delta E_{\mathrm{Ph}}$ and the strength of EPC contribution $F^{\mathrm{EPC}}$. The overall agreement is substantially improved. An exciton binding energy of $56 \mathrm{meV}$ is extracted, which is almost the same as that for $\mathrm{ZnO}$. It should be noticed the fitted energy of the exciton line is now approximately $13 \mathrm{meV}$ below the peak in $\varepsilon_{2}$, which is essential for the discussion of transition energies and comparison with previous studies. The same observation is made for all other samples except for the sample with $x=0.23$. Its broadening parameter of about $160 \mathrm{meV}$ does not allow separation of excitonic and EPC contributions. Finally, the results for $\mathrm{Mg}_{0.01} \mathrm{Zn}_{0.99} \mathrm{O}$ shown in Fig. 4(c) emphasize the accuracy of the extended DF model for fitting transition energies from $\mathrm{PbP}$ data; only the inclusion of EPC can explain the measured spectral dependence.

One can now proceed with the discussion of the fit results. For all samples with a $\mathrm{Mg}$ content up to $x=0.06$, an exciton binding energy of $56 \pm 3 \mathrm{meV}$ is obtained. It is about $3 \mathrm{meV}$ lower than for $\mathrm{ZnO}$, which is reasonable because the two contributions from $\mathrm{FX}_{\mathrm{A}}$ and $\mathrm{FX}_{\mathrm{B}}$ were replaced here by a single line with an averaged transition energy, whereas the onset of the exciton continuum at $E_{\mathrm{G}}$ remains unchanged. For higher $\mathrm{Mg}$ concentrations $(x=0.09,0.14$, and 0.23$), E_{\mathrm{B}}^{\mathrm{X}}$ continuously increases to 59,62 , and $65 \mathrm{meV}$, respectively. 

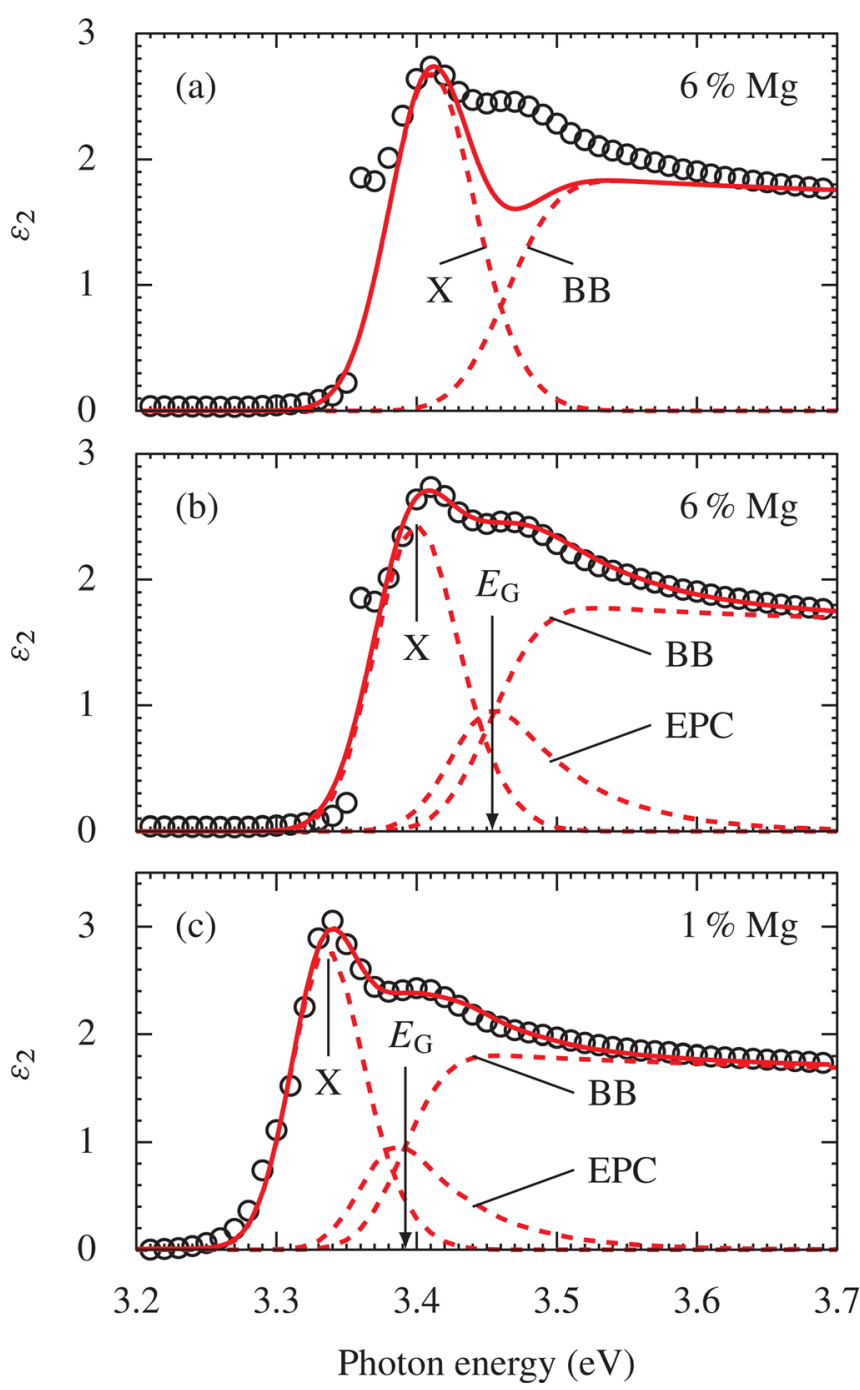

FIG. 4. (Color online) Imaginary part of the PbP DF (circles) and the fitted model dielectric function (solid line) around the bandgap for (a) $6 \% \mathrm{Mg}$, including only contributions (dashed lines) of free excitons (X) and band-toband transitions (BB); for (b) $6 \% \mathrm{Mg}$ additionally including transitions into EPC; and for (c) $1 \% \mathrm{Mg}$ including all the contributions. The bandgap energy $E_{\mathrm{G}}$ is marked by an arrow. All data are recorded at $T=295 \mathrm{~K}$. The discontinuity on the low energy side of (a) and (b) results from the fitting process and has no physical meanigful relevance.

This result deviates from the findings in Ref. 20 (fit without EPC), where a decrease of the exciton binding energy to 50 meV for $x=0.17$ and a subsequent re-increase was reported.

Figures 5(a) and 5(b) show the obtained values of $\Delta E_{\mathrm{Ph}}$ and $F^{\mathrm{EPC}}$, respectively. Our values of $\Delta E_{\mathrm{Ph}}$ range from 45 to $58 \mathrm{meV}$ with an average value of $51 \mathrm{meV}$. For comparison, the fit of $\mathrm{ZnO}$ in Refs. 32 and 18 yielded $55 \mathrm{meV}$ and 32 $\mathrm{meV}$, respectively. The $F^{E P C}$ values range from 0.54 to 0.91 , with an average value of 0.65 , being lower than the value of 0.99 reported in Ref. 18 but higher than 0.48 reported in Ref. 32. The low number of reported data so far does not allow us to provide a detailed evaluation here. It might be possible that the values depend as well on the structural properties of the samples.

In general, the values of $F^{\mathrm{EPC}}$ can be interpreted as the EPC enhancement factor, similar to the Sommerfeld enhancement factor for describing the exciton continuum. The significant influence on the DF of MgZnO cannot be dis-

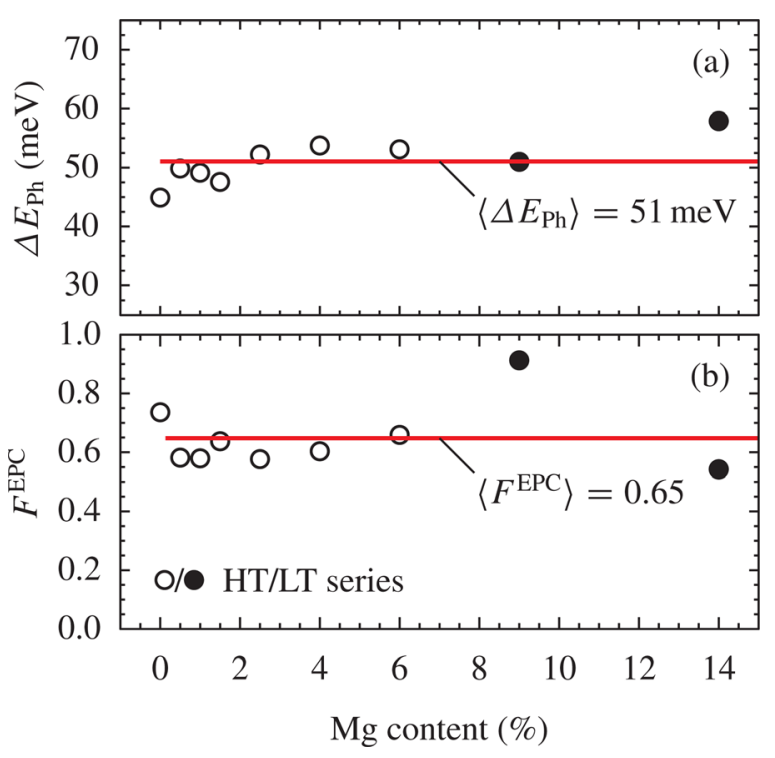

FIG. 5. (Color online) Extracted average phonon energy $\Delta E_{\mathrm{Ph}}$ (a) and contribution ratio $F^{E P C}$ (b) as a function of $\mathrm{Mg}$ concentration. The average values are depicted by the solid (red) lines.

regarded when modeling the spectra. The comparison of fit results without and with EPC reveals different transition energies for the excitonic lines.

For the interpretation of $\Delta E_{\mathrm{Ph}}$ the longitudinal-optical phonon energy $E^{\mathrm{LO}}$ has to be considered, which is about 72 $\mathrm{meV}$ for $\mathrm{ZnO}$ and increases slightly when alloying with Mg. ${ }^{35}$ Shokhovets et al. explained their too small $\Delta E_{\mathrm{Ph}}$ (therein denoted as $\Delta E$ ) as compared to $E_{\mathrm{ZnO}}^{\mathrm{LO}}$ with the participation of the various optical phonons of $\mathrm{ZnO}$ in the polarization cloud around the exciton. ${ }^{18}$ For GaN for example, they found a value of $\Delta E_{\mathrm{Ph}}$ only slightly lower than the corresponding $E_{\mathrm{GaN}}^{\mathrm{LO}}$, which therefore means the phonon cloud around the excitons is mainly formed by LO phonons. On the other hand, the room temperature absorption spectra of Liang and Yoffe ${ }^{9}$ show distinct EPC separated by multiples of $E_{\mathrm{ZnO}}^{\mathrm{LO}}$ from the main excitonic peak. This is also found at low temperatures ${ }^{9}$ and supported by the fitting results in Ref. 19. Respective separations are found for the first sideband in $\mathrm{GaN}^{13,14}$ or $\mathrm{MgO}$ and $\mathrm{BeO} .{ }^{11}$ Therefore we suggest the apparently smaller values of $\Delta E_{\mathrm{Ph}}$ derive from the facts that the line shape of the individual EPC are not considered and that the individual EPC contributions are not resolved in the room temperature DF published in Ref. 18. This should not belittle the models of Shokhovets et al. ${ }^{18}$ and Müller et al., ${ }^{19}$ as these are the only approaches permitting a parametric description of the room temperature DF. However, $\Delta E_{\mathrm{Ph}}$ should be considered only as a fitting parameter rather than a meaningful physical quantity.

\section{B. Temperature-dependent photoluminescence spectroscopy}

Photoluminescence spectroscopy is applied for further evaluation of the transition energies obtained from the evaluation of the DF, including EPC. Temperature-dependent studies yield the free excitonic transition energy at RT. Figures 6(a) and 6(b) show the PL spectra at $T=5 \mathrm{~K}$ for the HT 
and LT series, respectively, on a logarithmic scale. Characteristic features are denoted and connected by dashed lines as a guide to the eye. In order to analyze the data, first the $\mathrm{ZnO}$ spectrum is compared to published results summarized in a review article by Meyer et al $^{36}$ The main peak at 3.356 $\mathrm{eV}$ is located less than $1 \mathrm{meV}$ from the so called $I_{9}$ line at $3.3567 \mathrm{eV}$, which originates from recombinations of neutral donor bound excitons at the substitutional shallow donor indium. This is in agreement with the fact that In is used to mount the samples onto the substrate holder during MBE growth, thus being the main trace impurity. Therefore, the main peak is assigned to the $I_{9}$ line. Consequently, the two transitions located $71 \mathrm{meV}$ and $143 \mathrm{meV}$ below the $I_{9}$ peak correspond to multiples of the longitudinal-optical phonon energies in $\mathrm{ZnO}$ of around $72 \mathrm{meV}$ (Ref. 35) and are attrib-

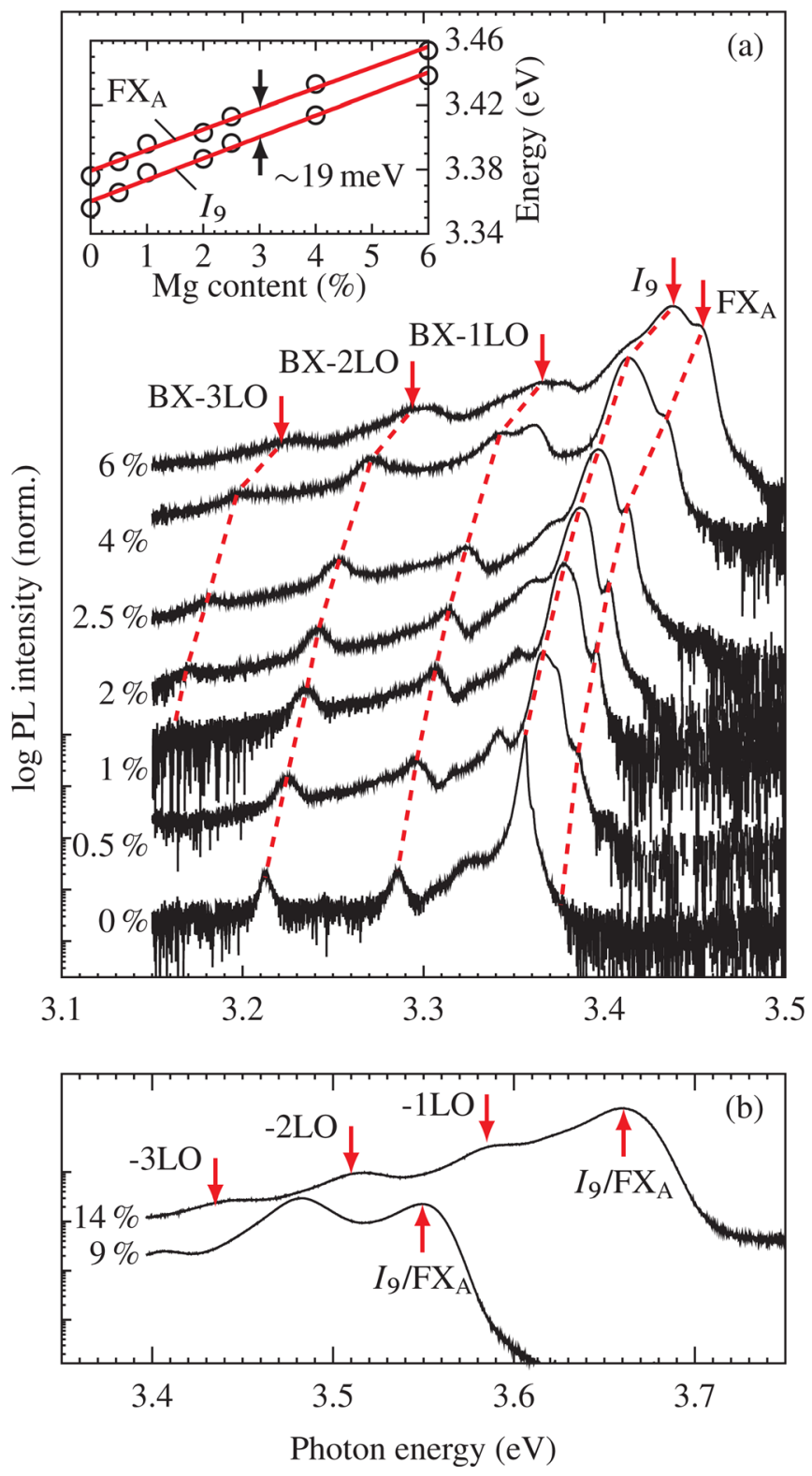

FIG. 6. (Color online) Normalized PL spectra of the (a) HT and (b) LT series at $T=5 \mathrm{~K}$ in logarithmic scale. The dashed lines show the transitions of the free exciton $\left(\mathrm{FX}_{\mathrm{A}}\right)$, the $I_{9}$ line, and its first three corresponding phonon replica (BX-LO). The inset of (a) shows the $I_{9}$ and $\mathrm{FX}_{\mathrm{A}}$ transitions vs the $\mathrm{Mg}$ content (circles) and the corresponding fits (red solid lines).

uted to phonon replicas of the main peak (bound excitons (BX)-LO). On the high energy side of the $I_{9}$ recombination a shoulder is located $19 \mathrm{meV}$ higher, at $3.376 \mathrm{eV}$, and identified as the free $\mathrm{A}$ exciton recombination $\left(\mathrm{FX}_{\mathrm{A}}\right)$. The transition energies for $I_{9}$ and $\mathrm{FX}_{\mathrm{A}}$ deviate less than $1 \mathrm{meV}$ from the results reported for bulk $\mathrm{ZnO}$. It can be emphasized that the film on the $\mathrm{MgO} / \mathrm{ZnO}$ buffer is almost strain-free within the light escape depth.

Similar to $\mathrm{ZnO}$, the $\mathrm{MgZnO}$ spectra of the HT series in Fig. 6(a) show a main peak and a high energy shoulder. The main differences are the shift toward higher energies and the broadening of the transitions due to alloying with $\mathrm{Mg}$. In the inset of Fig. 6(a) the transition energies of the peaks and the shoulders are plotted versus the Mg content for the HT samples. An almost constant energy difference of $19 \mathrm{meV}$ is found between these two transitions. As a result of the application of In during growth for all samples, it is concluded that the main peak is the corresponding $I_{9}$ line for $\mathrm{MgZnO}$ and that the high energy shoulder originates from recombinations of $\mathrm{FX}_{\mathrm{A}}$. For the LT series the alloy broadening of the transitions does not allow distinction of $I_{9}$ and $\mathrm{FX}_{\mathrm{A}}$ line as depicted in Fig. 6(b).

In order to determine the $\mathrm{FX}_{\mathrm{A}}$ transition energies at room temperature the temperature dependence of the PL spectra was examined. This is shown in Figs. 7(a) and 7(b) for the samples with $0.5 \%$ and $6 \% \mathrm{Mg}$ content, respectively. With increasing temperature the spectra are red shifted due to phonon-phonon and electron-phonon interaction, whereas the bound exciton transition disappears as a result of thermal dissociation. Therefore the spectra at temperatures above $110 \mathrm{~K}$ are dominated by the $\mathrm{FX}_{\mathrm{A}}$ transitions and their

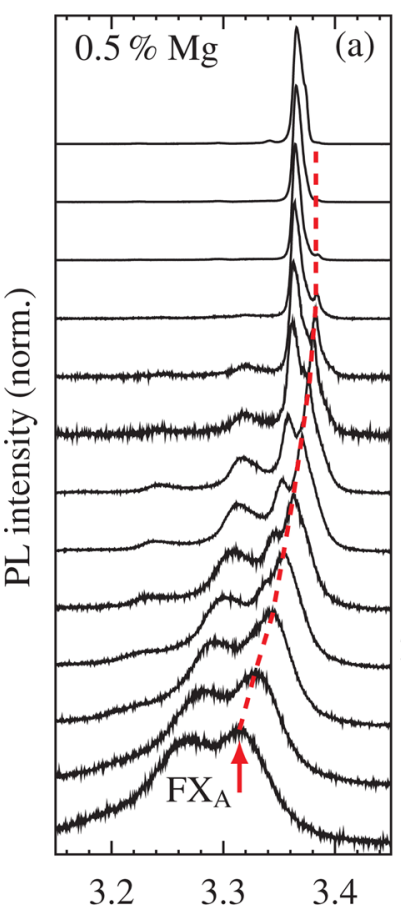

Photon energy (eV)

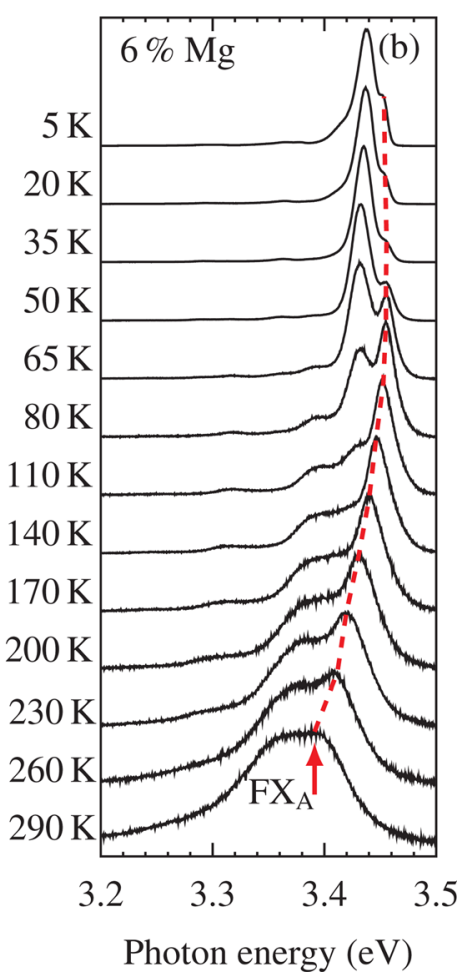

FIG. 7. (Color online) Normalized temperature-dependent PL spectra for the samples with $0.5 \%$ and $6 \% \mathrm{Mg}$ content. The dashed (red) lines show the change of the $\mathrm{FX}_{\mathrm{A}}$ transition energy. 
phonon replica, finally leading to broad weakly structured bands at room temperature (shown in Fig. 3 for all investigated samples). All spectra were fitted for all temperatures using peak functions to obtain accurate values for the $\mathrm{FX}_{\mathrm{A}}$ transition energies at room temperature. For the HT series Lorentz-shaped peak functions were used, whereas the LT samples were fitted using Gaussian-shaped peak functions due to the strong alloy broadening. As an example, the line shape fit for the sample with $6 \% \mathrm{Mg}$ concentration is shown in Fig. 8 for a temperature of $110 \mathrm{~K}$. Deviations occur in the region of the phonon replicas and on the high energy side of the $\mathrm{FX}_{\mathrm{A}}$ transition. The former originates mainly from the simple Lorentz line shape, which was used to model the data and the latter can also be assigned to reabsorption. A more elaborate fitting procedure taking into account the exciton distribution after the thermalization process and the density of states yielding a more accurate phonon replica line shape is found, e.g., in Ref. 37.

\section{Comparison of emission and absorption energies}

Figure 3 shows the comparison of emission and absorption properties in terms of room temperature PL spectra and $\varepsilon_{2}$. We do not find a pronounced splitting between the emission and absorption spectra up to $x=0.06$. This finding is also emphasized by the data in Table I, where a summary of the fitted transition energies from PL at 5 and $295 \mathrm{~K}$ as well as of the $F X_{\mathrm{X}}^{\mathrm{SE}}$ data (with EPC inclusion) at $\mathrm{RT}$ is presented. The emission and absorption data of $\mathrm{ZnO}$ for example deviate by only $2 \mathrm{meV}$. It proves the accuracy of the DF model with EPC. Hence, the splitting between $F X_{\mathrm{A}}^{\mathrm{PL}}$ from PL and $F X_{\mathrm{X}}^{\mathrm{SE}}$ at $T=295 \mathrm{~K}$ can be considered as measure for the Stokes shift. This difference is depicted in the inset of Fig. 3, the dependence on the $\mathrm{Mg}$ concentration is attributed to potential fluctuations of the bandgap caused by spatially inhomogeneous alloy composition. SE determines the average exciton transition energy, while in PL transitions occur after relaxation to potential minima, yielding a lower recombination energy. This is confirmed by the fact that for the HT series this shift is less than $15 \mathrm{meV}$, while for the LT series

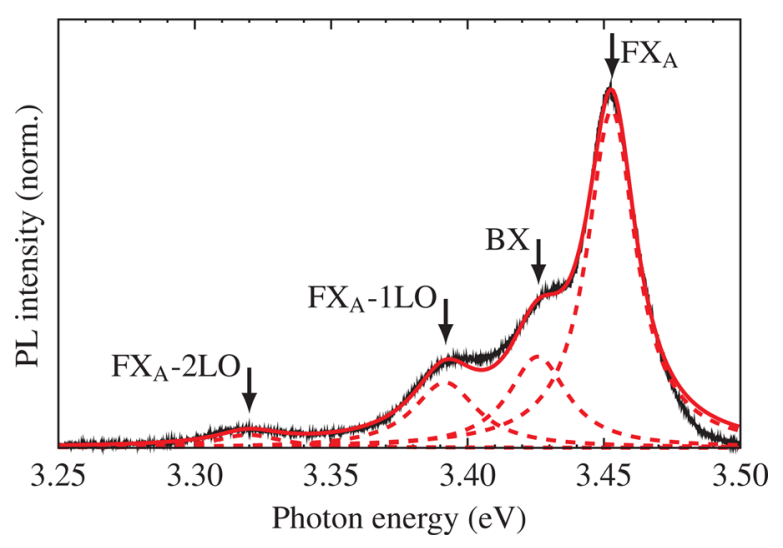

FIG. 8. (Color online) PL spectrum of the $6 \% \mathrm{Mg}$ sample at $110 \mathrm{~K}$. The thick (black) line shows the experimental results, and the thin (red) line the fit. Contributions of free $\left(\mathrm{FX}_{\mathrm{A}}\right)$ and bound excitons $(\mathrm{BX})$ and the first two LO phonon replicas of FX are shown as dashed lines. more than $45 \mathrm{meV}$ is found. In addition, the LT series SE spectra are significantly broadened, suggesting a stronger alloy-dependent disorder due to the lower growth temperature.

The microscopic potential minima leading to lower transition energies in PL experiments compared to SE can be explained as either related to random alloying or to the creation of clusters. In a recently published approach, Grundmann and Dietrich ${ }^{38}$ try to distinguish between these two possibilities by luminescence line shape analysis. The full width at half maximum (FWHM) measured by PL can be described by

$$
\mathrm{FWHM} \approx 2 \sqrt{2 \ln 2} \frac{\delta E_{\mathrm{FX}}}{\delta x} \sqrt{\frac{x(N-x)}{K V_{\mathrm{exc}}}},
$$

where $\delta E_{\mathrm{FX}} / \delta x$, the dependence of the free exciton energy on the $\mathrm{Mg}$ content $x$, is available from our SE data (Table I). $K$ is the cation concentration, and $V_{\text {exc }}$ the volume of the exciton. $N$ can be understood as the average number of $\mathrm{Mg}$ atoms bound to the same oxygen atom. For low Mg content, this number is expected to be $N=1$. If clustering of $\mathrm{Mg}$ atoms occur, $N$ can increase up to $N=4$.

In Fig. 9 our measured FWHM values are compared with the results of Eq. (5). At low temperature, the FWHM values of samples from the HT series (circles) are within error margins well reproduced by Eq. (5) using $N=1$. For FWHM values measured from the LT series (dots) samples, $N=4$ has to be used to reproduce the experimental values, indicating that clustering of $\mathrm{Mg}$ atoms occurs in these layers. By increasing the lattice temperature, i.e., measuring the FWHM at room temperature, excitons can leave local potential minima and would end up in energetically more favorable states, i.e., lower energy. To account for elevated temperatures, the calculated FWHM should be increased by a temperature-dependent value dominated by longitudinal optical phonon broadening. In Ref. 38, fitting of temperature-dependent data yields a value of $2 \sqrt{2 \ln 2 \Gamma}=46 \mathrm{meV}$, which we adopt here. Reasonable agreement is obtained for FWHM values at $T=295 \mathrm{~K}$, as is shown in Fig. 9. As

TABLE I. Transition energies (in eV) determined by fitting the experimental data.

\begin{tabular}{lccccc}
\hline \hline & \multicolumn{2}{c}{$T=5 \mathrm{~K}$} & & \multicolumn{2}{c}{$T=295 \mathrm{~K}$} \\
\cline { 2 - 3 } \cline { 5 - 6 } Mg content $(\%)$ & $F X_{\mathrm{A}}^{\mathrm{PL}}$ & $I_{9}^{\mathrm{PL}}$ & & $F X_{\mathrm{A}}^{\mathrm{PL}}$ & $F X_{\mathrm{X}}^{\mathrm{SE}}$ \\
\hline 0 & 3.376 & 3.356 & 3.308 & 3.310 \\
0.5 & 3.385 & 3.366 & 3.316 & 3.320 \\
1.0 & 3.396 & 3.378 & 3.328 & 3.335 \\
1.5 & 3.402 & 3.387 & 3.339 & 3.343 \\
2.5 & 3.413 & 3.396 & 3.347 & 3.357 \\
4 & 3.433 & 3.414 & 3.362 & 3.377 \\
6 & 3.453 & 3.438 & 3.397 & 3.398 \\
9 & 3.549 & - & 3.488 & 3.536 \\
14 & 3.661 & - & 3.615 & 3.669 \\
23 & - & - & - & 3.915 \\
\hline \hline
\end{tabular}




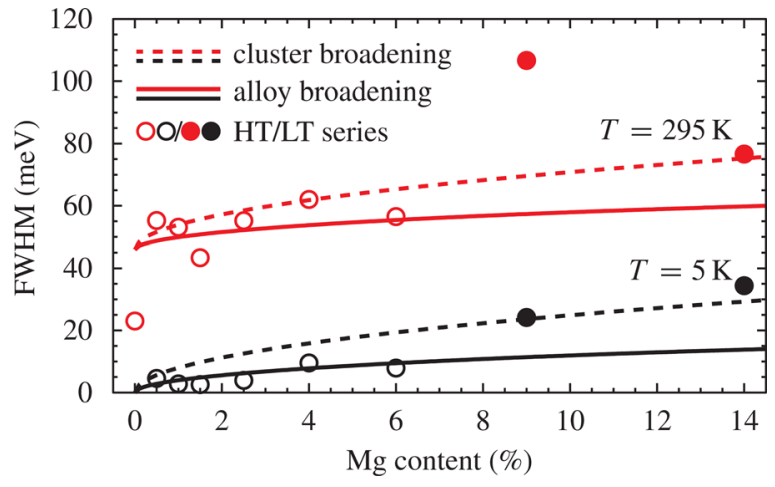

FIG. 9. (Color online) Full width at half maximum (FWHM) of the free exciton emission at $T=5 \mathrm{~K}$ (black) and at room temperature (red) in comparison to model calculations accounting for random alloying (solid lines) and clustering (dashed lines). The difference between FWHM at $5 \mathrm{~K}$ and RT is well described by a constant offset of $46 \mathrm{meV}$, as calculated in Ref. 38 .

expected, data points from both growth series follow the predicted behavior of random alloys within error margins.

Comparing the low temperature PL FWHM values to the Stokes shift between SE and PL (at room temperature, shown as inset in Fig. 3) we find good agreement of both dependences on $x$. This supports our finding that disorder of the ternary alloy has to be taken into account when analyzing optical data of $\mathrm{Mg}_{\mathrm{x}} \mathrm{Zn}_{1-\mathrm{x}} \mathrm{O}$ samples. The PL FWHM at low temperatures, representing the disorder within the laser excitation spot, follows the same behavior as the Stokes shift at room temperature, which takes account of the difference between averaged $\mathrm{Mg}$ concentration and potential minima where emission favorably takes place. The characteristic localization energy of each sample can be evaluated by the Stokes shift between SE and PL at room temperature or by the FWHM of PL at low temperature. The Stokes shift is found to be about twice the value of the FWHM at $5 \mathrm{~K}$. For the sample with $x=0.14$ this value is $51 \mathrm{meV}$, in remarkable agreement with an earlier study, ${ }^{39}$ where about $60 \mathrm{meV}$ for $x \approx 0.21$ was evaluated as a characteristic localization energy.

\section{CONCLUSION}

The imaginary part of the dielectric function of $\mathrm{MgZnO}$ was modeled with high accuracy including electron-hole interaction and exciton-phonon complexes. The analysis yields reliable excitonic transition energies, which are emphasized by the line shape of the PL spectra. The fitting parameters for the exciton-phonon complexes show almost constant and significant contributions of exciton-phonon complexes for $\mathrm{Mg}$ contents less than 23\%. The values of $\Delta E_{\mathrm{Ph}}$ do not correspond to the longitudinal-optical phonon energies nor do they represent a real physical quantity. The Stokes shift can be attributed to clustering of $\mathrm{Mg}$ atoms at low growth temperatures.

${ }^{1}$ C. Klingshirn, Phys. Status Solidi B 244, 3027 (2007).

${ }^{2}$ D. C. Reynolds, D. C. Look, B. Jogai, C. W. Litton, G. Cantwell, and W. C. Harsch, Phys. Rev. B 60, 2340 (1999).
${ }^{3}$ S. F. Chichibu, A. Uedono, A. Tsukasaki, T. Onuma, M. Zamfirescu, A. Ohtomo, A. Kavokin, G. Cantwell, C. W. Litton, T. Sota, and M. Kawasaki, Semicond. Sci. Technol. 20, S67 (2005).

${ }^{4}$ A. Ohtomo, M. Kawasaki, T. Koida, K. Masubuchi, H. Koinuma, Y. Sakurai, Y. Yoshida, T. Yasuda, and Y. Segawa, Appl. Phys. Lett. 72, 2466 (1998).

${ }^{5}$ P. Gori, M. Rakel, C. Cobet, W. Richter, N. Esser, A. Hoffmann, R. D. Sole, A. Cricenti, and O. Pulci, Phys. Rev. B 81, 125207 (2010).

${ }^{6}$ A. Schleife, C. Rödl, F. Fuchs, J. Furthmüller, F. Bechstedt, P. H. Jefferson, T. D. Veal, C. F. McConville, L. F. J. Piper, A. DeMasi, K. E. Smith, H. Lösch, R. Goldhahn, C. Cobet, J. Zúñiga-Pérez, and V. Muñoz-Sanjosé, J. Korean Phys. Soc. 53, 2811 (2008).

${ }^{7}$ R. J. Elliott, Phys. Rev. 108, 1384 (1957).

${ }^{8}$ G. E. Jellison and L. A. Boatner, Phys. Rev. B 58, 3586 (1998).

${ }^{9}$ W. Y. Liang and A. D. Yoffe, Phys. Rev. Lett. 20, 59 (1968).

${ }^{10}$ M. Cobet, C. Cobet, M. R. Wagner, N. Esser, C. Thomsen, and A. Hoffmann, Appl. Phys. Lett. 96, 031904 (2010).

${ }^{11}$ W. C. Walker, D. M. Roessler, and E. Loh, Phys. Rev. Lett. 20, 847 (1968).

${ }^{12}$ J. Dillinger, Č. Koňák, V. Prosser, J. Sak, and M. Zvára, Phys. Status Solidi B 29, 707 (1968)

${ }^{13}$ A. J. Fischer, W. Shan, J. J. Song, Y. C. Chang, R. Horning, and B. Goldenberg, Appl. Phys. Lett. 71, 1981 (1997).

${ }^{14}$ J. F. Muth, J. H. Lee, I. K. Shmagin, R. M. Kolbas, H. C. Casey, B. P. Keller, U. K. Mishra, and S. P. DenBaars, Appl. Phys. Lett. 71, 2572 (1997).

${ }^{15}$ J. Sak, Phys. Rev. Lett. 25, 1654 (1970).

${ }^{16}$ R. Zimmermann and C. Trallero-Giner, Phys. Rev. B 56, 9488 (1997).

${ }^{17}$ K. Hannewald and P. A. Bobbert, Phys. Rev. B 72, 113202 (2005).

${ }^{18} \mathrm{~S}$. Shokhovets, O. Ambacher, B. K. Meyer, and G. Gobsch, Phys. Rev. B 78, 035207 (2008).

${ }^{19}$ A. Müller, G. Benndorf, S. Heitsch, C. Sturm, and M. Grundmann, Solid State Commun. 148, 570 (2008).

${ }^{20}$ R. Schmidt, B. Rheinländer, M. Schubert, D. Spemann, T. Butz, J. Lenzner, E. M. Kaidashev, M. L. A. Rahm, H. C. Semmelhack, and M. Grundmann, Appl. Phys. Lett. 82, 2260 (2003).

${ }^{21}$ A. Bakin, A. El-Shaer, A. C. Mofor, M. Kreye, A. Waag, F. Bertram, J. Christen, M. Heuken, and J. Stoimenos, J. Cryst. Growth 287, 7 (2006).

${ }^{22}$ T. A. Wassner, B. Laumer, S. Maier, A. Laufer, B. K. Meyer, M. Stutzmann, and M. Eickhoff, J. Appl. Phys. 105, 023505 (2009).

${ }^{23}$ A. Schleife, F. Fuchs, J. Furthmüller, and F. Bechstedt, Phys. Rev. B 73, 245212 (2006).

${ }^{24}$ A. Schleife, C. Rödl, F. Fuchs, J. Furthmüller, and F. Bechstedt, Appl. Phys. Lett. 91, 241915 (2007).

${ }^{25}$ M. Schubert, B. Rheinländer, J. A. Wollam, B. Johs, and C. M. Herzinger, J. Opt. Soc. Am. A 13, 875 (1996).

${ }^{26}$ S. Shokhovets, L. Spieß, and G. Gobsch, J. Appl. Phys. 107, 023509 (2010).

${ }^{27}$ D. E. Aspnes, J. Opt. Soc. Am. 70, 1275 (1980).

${ }^{28}$ R. Goldhahn, Acta Phys. Pol. A 104, 123 (2003).

${ }^{29}$ H. Yao and C. H. Yan, J. Appl. Phys. 85, 6717 (1999).

${ }^{30}$ D. A. G. Bruggeman, Anal. Phys. 416, 636 (1935).

${ }^{31}$ M. R. Wagner, J.-H. Schulze, R. Kirste, M. Cobet, A. Hoffmann, C. Rauch, A. V. Rodina, B. K. Meyer, U. Röder, and K. Thonke, Phys. Rev. B 80, 205203 (2009).

${ }^{32} \mathrm{~S}$. Shokhovets, G. Gobsch, and O. Ambacher, Superlattices Microstruc. 39, 299 (2006).

${ }^{33}$ Q. Xu, X.-W. Zhang, W.-J. Fan, S.-S. Li, and J.-B. Xia, Comput. Mater. Sci. 44, 72 (2008).

${ }^{34}$ A. Schleife, F. Fuchs, C. Rödl, J. Furthmüller, and F. Bechstedt, Phys. Status Solidi B 246, 2150 (2009).

${ }^{35}$ C. Bundesmann, A. Rahm, M. Lorenz, M. Grundmann, and M. Schubert, J. Appl. Phys. 99, 113504 (2006).

${ }^{36}$ B. K. Meyer, H. Alves, D. M. Hofmann, W. Kriegseis, D. Forster, F. Bertram, J. Christen, A. Hoffmann, M. Straßburg, M. Dworzak, U. Haboeck, and A. V. Rodina, Phys. Status Solidi B 241, 231 (2004).

${ }^{37}$ R. Hauschild, H. Priller, M. Decker, J. Brückner, H. Kalt, and C. Klingshirn, Phys. Status Solidi C 3, 976 (2006).

${ }^{38}$ M. Grundmann and C. P. Dietrich, J. Appl. Phys. 106, 123521 (2009).

${ }^{39}$ A. Chernikov, S. Horst, M. Koch, K. Volz, S. Chatterjee, S. W. Koch, T. A. Wassner, B. Laumer, and M. Eickhoff, J. Lumin. 130, 2256 (2010). 